\title{
A Comparative Study of Location Aided Routing Protocols for MANET
}

\author{
Vasil Hnatyshin, Malik Ahmed, Remo Cocco, and Dan Urbano \\ Department of Computer Science \\ Rowan University \\ Glassboro, NJ 08028
}

\begin{abstract}
Location-aided routing (LAR) is a mechanism which attempts to reduce the control message overhead of Ad-hoc ondemand distance vector (AODV) routing protocol by flooding only the portion of the network that is likely to contain the route to destination. LAR takes advantage of Global Positioning System (GPS) coordinates to identify a possible location of the destination node. Based on this information, LAR defines a portion of the network which will be subject to the limited flooding, thus reducing the total number of the control packet traveling through the network during the route discovery process. GeoAODV is a variation of the AODV protocol which like LAR also employs GPS coordinates to limit the search area used during the route discovery process. However, unlike LAR, GeoAODV does not make the assumption that every node in the network knows the traveling speed and location of the corresponding destination node. Instead, GeoAODV tries to dynamically learn and distribute location information among the nodes in the network. This paper examines and compares through simulation the performance of AODV, LAR, and GeoAODV protocols under different environmental settings.
\end{abstract}

Keywords-Ad-hoc on-demand distance vector; Location Aided Routing; Geographical AODV; Mobile Ad-hoc Networks;

\section{INTRODUCTION}

Ad-hoc on-demand distance vector (AODV) is a reactive routing protocol for mobile ad-hoc networks (MANETs) [1, 2]. AODV, as the name implies, is a distance-vector routing protocol that uses the hop count, or the number of nodes in a route, to discover the best routes between the nodes in the network. AODV attempts to discover a route to destination only when it is needed, i.e., a source node has data to be delivered to destination but a route to destination is unknown. A source node initiates the route discovery process by broadcasting a route request (RREQ) packet to all of its neighbors. The nodes that receive RREQ packets re-broadcast them in the network until the destination or a node that knows the path to destination is found. Once an RREQ message arrives at the destination or at a node that knows a path to the destination, a route reply (RREP) message is generated and unicast back to the source node. Arrival of RREP message at the source indicates a completion of route discovery process, at which point the data packets are forwarded to the destination using unicast transmission.

The AODV route discovery process also employs such mechanisms as sequence numbers and hop count to prevent a node from forwarding the same RREQ twice and to limit how far the request messages will travel in the searched MANET domain
[3]. This route discovery technique is referred to as flooding and it is often employed because of its simplicity.

Location Aided Routing (LAR) is one of the most popular mechanisms for reducing control message overhead in AODV [7, 8]. LAR assumes that the nodes in the network know their own location and can obtain the last known position of the destination node. Based on this information, LAR limits the route search area to the portion of the network where the route to destination node is likely to be located. LAR modifies the route discovery process so that only the nodes which belong to the search area would rebroadcast RREQ messages. Specifically, when an RREQ packet arrives at an intermediate node, the node first determines whether it belongs to the search area defined by the incoming RREQ message. If the node is part of that search area then the RREQ packet is rebroadcasted, otherwise the packet is discarded.

Geographical AODV (GeoAODV) is another variation of AODV protocol which like LAR limits the search area to the portion of the network which is likely to contain the route to destination [9, 10]. However, unlike LAR, GeoAODV does not assume that the destination coordinates are available to a node which initiates the route discovery process. GeaAODV relies on a distributed process to share location coordinates among the nodes in the network. In addition, GeoAODV defines the search area differently than LAR and it can dynamically adjust the search area as the RREQ messages traverse the network.

In this paper, we discuss the results of a new comprehensive simulation study, conducted using OPNET Modeler version 16.0 software package [11], in which we compare the performance of AODV and four location-aided routing protocols (i.e., two variations of LAR and two variations of GeoAODV). In section two we provide an overview of AODV, LAR, and GeoAODV protocols. Section three describes the simulation set-up, followed by section four which presents and analyzes the results of our simulation study. We conclude in section five.

\section{OVERVIEW OF COMPARED PROTOCOLS}

\section{a. Location Aided Routing}

Over the last few years, there have been numerous variations of LAR protocol examined in the literature [12-17]. However, in our study we compare the performance of the original LAR scheme 1 and LAR scheme 2 studied in $[7,8]$.

LAR scheme 1, which we will call LAR zone, operates as follows. LAR assumes that each node in the network knows the destination's physical location at time $t_{0}$ as well as its average 
traveling speed $v$. Based on this information, the source node computes the destination's expected zone at time $t_{l}$ as a circle centered in the destination's last know position and radius $r$ which is computed as follows:

$$
r=v \times\left(t_{1}-t_{0}\right)
$$

The expected zone is the area where the destination node is expected to be located at time $t_{l}$. LAR zone defines the destination's request zone as an area which is likely to contain the path to destination. The request zone is defined as a rectangular area with the following properties: (1) the request zone completely encompasses the expected area, (2) the sides of the rectangle are parallel to the $x$ and y coordinates, and (3) the sides of the rectangle are tangent to the expected zone circle. Figure 1(a) illustrates the definition of expected and request zones for the LAR zone protocol. During the route discovery process only the nodes within the destination's request zone participate in finding the path to destination by re-broadcasting RREQ messages.

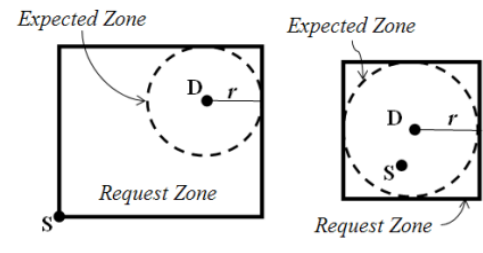

(a)

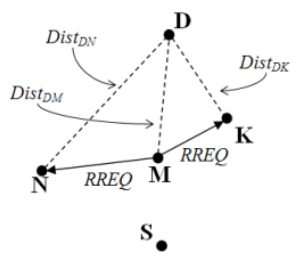

(b)
Figure 1. LAR examples: (a) LAR zone (b) LAR distance

LAR scheme 2, which we will call LAR distance, relies on the distance between the intermediate node and the destination to determine if an RREQ message will be rebroadcast or not. During the route discovery process in LAR distance protocol, the intermediate node rebroadcasts the RREQ message only if the distance from the destination to this intermediate node, Dist $_{i}$, is shorter than the distance from the destination to the node which generated this RREQ (i.e., the previous hop), Dist $t_{p}$. Specifically, the intermediate node rebroadcasts the RREQ only if the equation 2 holds true ( $\alpha$ and $\beta$ are configuration parameters):

$$
\alpha \times \text { Dist }_{p}+\beta \geq \text { Dist }_{i}
$$

\section{b. GeoAODV}

GeoAODV $[9,10]$ is a variation of LAR which uses a cone shaped request area. Initially, GeoAODV assumes that each node only knows its own position and does not possess any knowledge about the destination's traveling speed or coordinates. The request zone in GeoAODV is defined by a flooding angle, which is evenly divided by a line that would connect the source and the destination. As shown in Figure 2(a), $\alpha$ is a flooding angle which defines the cone-shaped request zone. When an intermediate node $\mathbf{N}$ receives a route request message, it computes the angle $\theta$ formed between nodes $\mathbf{N}, \mathbf{S}$, and $\mathbf{D}$. If $S D$ is the vector from source to destination and $S N$ is the vector from the source to the node, then the node's angle, $\theta$, is computed as follows:

$$
\theta=\cos ^{-1}((S D \cdot S N) /(|S D| \times|S N|))
$$

If $\theta$ is less than half of the flooding angle $\alpha$ then node $\mathbf{N}$ is considered to be located within the request zone and will rebroadcast an RREQ message. Otherwise the message is discarded. In this paper, we will refer to this GeoAODV protocol as GeoAODV static.

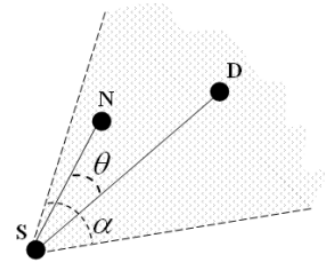

(a)

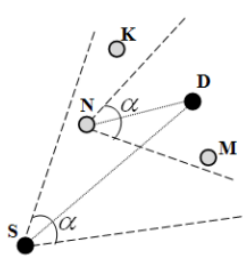

(b)
Figure 2. GeoAODV examples: (a) regular GeoAODV (b) GeoAODV rotate

GeoAODV rotate is a variation of GeoAODV static protocol. Instead of always using the same request zone throughout each round of the route discovery process, the cone-shaped area is dynamically adjusted at each intermediate node to better reflect possible location of the path to destination. Specifically, instead of always computing the request zone as a cone-shaped area formed with the source node at the cone's apex, each intermediate node computes the request zone as a cone-shaped area formed by having itself at the cone's apex as shown in. Figure 2(b).

TABLE I. SUMMARY OF NODE CONFIGURATION

\begin{tabular}{|l|l|}
\hline \multicolumn{1}{|c|}{ Configuration Parameter } & \multicolumn{1}{c|}{ Value } \\
\hline Channel Data Rate & $11 \mathrm{Mbps}$ \\
\hline Transmit Power & $0.001 \mathrm{Watts}$ \\
\hline Packet Reception Power Threshold & $-95 \mathrm{dBm}$ \\
\hline Start of data transmission* & normal $(100,5)$ seconds \\
\hline End of data transmission & End of simulation \\
\hline Packet inter-arrival time* & exponential $(1)$ second \\
\hline Packet size* & exponential $(1024)$ bytes \\
\hline
\end{tabular}

\section{Simulation SET-UP}

To compare the performance of the AODV, LAR, and GeoAODV protocols, we developed a simulation study using OPNET Modeler version 16.0 [12]. We implemented the LAR and GeoAODV protocols by modifying the standard AODV process model in OPNET [9]. LAR distance was configured to have $\alpha=1$ and $\beta=0$, while GeoAODV protocols had the flooding angle initially set to 90 degrees. The value of the flooding angle was increased to 180,270 , and 360 degrees after each unsuccessful route discovery attempt.

TABLE II. SUMMARY OF THE SIMULATION STUDY

\begin{tabular}{|c|c|}
\hline $\begin{array}{c}\text { No. of } \\
\text { communicating nodes }\end{array}$ & $\begin{array}{c}\text { Node traveling speed } \\
\text { (meters/second) }\end{array}$ \\
\cline { 1 - 2 } 2 & \multirow{2}{*}{$\begin{array}{c}\text { 0 (static) }, 1,5,10,20, \\
\text { Uniformly distributed } \\
\text { between }(0,20)\end{array}$} \\
\hline 5 & \\
\hline 20 & \\
\hline 30 & \\
\hline
\end{tabular}


The simulation study consisted of several scenarios each executed for 500 seconds. In each scenario we randomly placed 50 mobile nodes in the $1500 \times 1500$ meter area. Table I provides a summary of individual node configuration. The configuration parameters marked with an asterisk $(*)$ had their value computed using a probability distribution function with the function name and input parameter values specified in the value column. Table II provides a summary of the simulation scenarios examined in this study. Each simulation scenario was executed five times and the results were averaged over the number of runs.

\section{RESULTS}

Due to space limitations, we only include summary of the main simulation results. Figure 3 illustrates the adjusted control traffic overhead vs. the number of communicating nodes for scenarios when the node traveling speed was selected randomly. We computed the adjusted control traffic overhead as the total number of RREQ and RREP packet divided by the number communicating nodes.

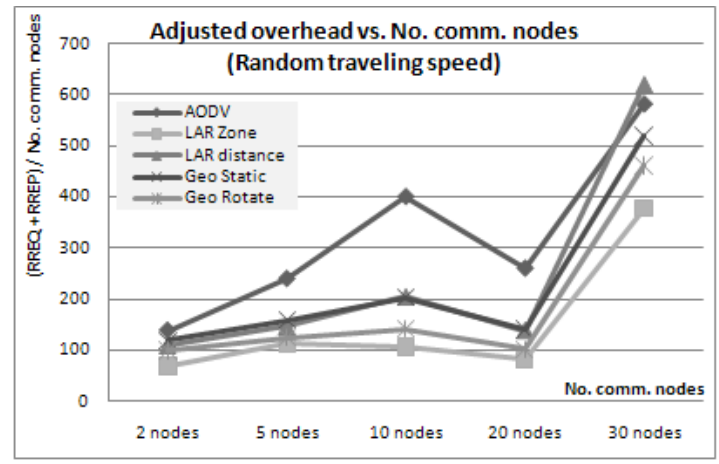

Figure 3. Adjusted RREQ and RREP overhead

As expected, collected simulation results reported that LAR zone and both variations of the GeoAODV protocol consistently generate fewer control packets than the regular AODV protocol. Overall, LAR zone consistently generated fewer RREQ and RREP messages than any other protocol. However, despite relying on the distributed protocol to obtain destination coordinates, GeoAODV rotate was a close second. GeoAODV rotate performed only slightly worse than LAR zone for majority of the simulation scenarios while consistently outperforming LAR distance and GeoAODV static protocols.

\section{CONCLUSIONS}

In this paper we presented the results of a comparison study of AODV, LAR, and GeoAODV protocols. As expected, simulation results suggest that location-aided routing protocols generate significantly fewer control RREQ and RREP messages than regular AODV protocol. Even though LAR zone consistently outperformed all the other protocols examined in this study, performance of GeoAODV rotate protocol was comparable. We believe that GeoAODV rotate deserves a closer examination because it employs a distributed protocol to find destination coordinates and it gradually reverts to regular AODV whereas LAR protocols assumes that destination node coordinates and traveling speed are known and it reverts to AODV after a single failure.

\section{REFERENCES}

[1] E. M. Royer and C. E. Perkins. "An Implementation Study of the AODV Routing Protocol," Proc. of the IEEE Wireless Communications and Networking Conference, Chicago, IL, September 2000.

[2] C. E. Perkins and E. M. Royer. "Ad hoc On-Demand Distance Vector Routing," Proc. of the 2nd IEEE Workshop on Mobile Computing Systems and Applications, New Orleans, LA, Feb. 1999, pp. 90-100.

[3] D. Espes, Z. Mammeri. "Adaptive expanding search methods to improve AODV Protocol," IST Mobile and Wireless Communications Summit, July 2005.

[4] Lee, S.-J., Belding-Royer, E. M. and Perkins, C. E. (2003), "Scalability study of the ad hoc on-demand distance vector routing protocol," International Journal of Network Management, 13: 97-114.

[5] S. M. Das, H. Pucha, and Y. C. Hu, "Performance comparison of scalable location services for geographic ad hoc routing," in Proc. IEEE 24th Annu. Joint Conf. IEEE Comput. Commun. Soc., 2005, pp. 1228-1239.

[6] E. Kuiper, S. Nadjm-Tehrani, "Geographical Routing With Location Service in Intermittently Connected MANETs," Vehicular Technology, IEEE Transactions on, vol.60, no.2, Feb. 2011, pp.592-604,

[7] Y. Ko and N. H. Vaidya, "Location-aided routing (LAR) in mobile ad hoc networks," Wireless Networks, 6(4), July 2000, pp. 307-321.

[8] Y. Ko and N. H. Vaidya, "Flooding-based geocasting protocols for mobile ad hoc networks," Mobile Networks and Applications, 7(6), Dec. 2002, pp. 471-480.

[9] V. Hnatyshin, and H. Asenov, "Design and Implementation of an OPNET model for simulating GeoAODV MANET routing protocol", Proc. of the OPNETWORK 2010 International Conference, Session: Wireless Ad Hoc and Wireless Personal Area Networks, Washington DC, August 2010.

[10] H. Asenov, and V. Hnatyshin, "GPS-Enhanced AODV routing," in Proceedings of the 2009 International Conference on Wireless Networks (ICWN'09), Las Vegas, Nevada, USA (July 13-16, 2009)

[11] OPNET Modeler ver. 16.0. OPNET Technologies, Inc $®$, www.opnet.com last visited 7/6/11.

[12] A. Husain, B. Kumar, A. Doegar, "A Study of Location Aided Routing (LAR) Protocol for Vehicular Ad Hoc Networks in Highway Scenario," International Journal of Engineering and Information Technology, 2(2), 2010, pp 118-124.

[13] K.M.E. Defrawy and G. Tsudik, "ALARM: Anonymous Location-Aided Routing in Suspicious MANETs," Proc. of the IEEE International Conference on Network Protocols, 2007, pp.304-313.

[14] D. Deb, S. B. Roy, N. Chaki, "LACBER: A new location aided routing protocol for GPS scarce MANET," International Journal of Wireless \& Mobile Networks (IJWMN), 1(1), August 2009.

[15] F. De Rango, A. Iera, A. Molinaro, S. Marano, "A modified location-aided routing protocol for the reduction of control overhead in ad-hoc wireless networks, " Proc. of the 10th International Conference on Telecommunications, 2003, pp. $1033-1037$.

[16] Y. Xue, B. Li, "A Location-aided Power-aware Routing Protocol in Mobile Ad Hoc Networks," Proc. of the IEEE Global Telecommunications Conference, San Antonio, TX, November 2001, pp. 2837 - 2841.

[17] Y. Wang, L. Dong, T. Liang, X. Yang, X., D. Zhang, "Cluster based location-aided routing protocol for large scale mobile ad hoc networks," IEICE Transactions, 2009, E92-D(5), pp. 1103-1124. 